\title{
Sistema de alerta temprana para previsión de avenidas en la ciudad de Durazno
}

\section{Early warning system for flood forecast in Durazno city}

\author{
Silveira, Luis ${ }^{(1)}$, Chreties, Christian ${ }^{(1)}$, Crisci, Magdalena (1), Usera, Gabriel (1), Alonso, Jimena ${ }^{(1)}$. \\ (1) Instituto de Mecánica de los Fluidos e Ingeniería Ambiental (IMFIA), Facultad de Ingeniería, Universidad de la República. \\ Contacto: lesy@fing.edu.uy
}

RECIBIDO: 13/3/2015 - APROBADO: 22/12/2015

\begin{abstract}
Resumen
Desde mayo de 2011, el Centro Coordinador de Emergencias Departamentales (CECOED) de Durazno cuenta con un Sistema de Alerta Temprana operativo para previsión de las avenidas del río Yí. Este sistema fue desarrollado en el marco de un proyecto de la red PROHIMET (financiado por la Organización Meteorológica Mundial-OMM) por el Instituto de Mecánica de los Fluidos e Ingeniería Ambiental (IMFIA)-Facultad de Ingeniería, con la coparticipación del Instituto de Teoría y Urbanismo (ITU)-Facultad de Arquitectura, la Dirección Nacional de Aguas (DINAGUA-MVOTMA), el Sistema Nacional de Emergencias (SINAE), la Administración Nacional de Usinas y Transmisiones Eléctricas (UTE), el Instituto Nacional de Meteorología (INUMET), y el Ministerio de Ganadería, Agricultura y Pesca (MGAP). En este trabajo se describen las principales características del Sistema de Alerta Temprana instalado y se presenta la metodología empleada, información de base y cómo ha sido su desempeño y eficacia en la gestión de las emergencias frente a tormentas extremas de diferentes características ocurridas entre los años 2011 a 2014.
\end{abstract}

Palabras clave: Inundaciones, emergencias climáticas, modelación hidrológica.

\begin{abstract}
Since May 2011, the Coordination Center of Departmental Emergencies (CECOED) of Durazno City has an Early Warning System to forecast floods of the Yí river. This system was developed as part of the PROHIMET project (funded by the World Meteorological Organization) by the Institute of Fluid Mechanics and Environmental Engineering (IMFIA), Faculty of Engineering, and the inputs of the Institute of Theory and Urban Development (ITU), Faculty of Architecture, the National Water Directorate (DINAGUA-MVOTMA), the National Emergency System (SINAE), the National Administration of Electric Power Plants (UTE), the National Institute of Meteorology (INUMET), and the Ministry of Livestock, Agriculture and Fisheries (MGAP). This paper describes the main characteristics of the Early Warning System and the methodology carried out, the input data and its performance and effectiveness regarding the operational management of emergencies caused by extreme storms with different climatic characteristics occurred between 2011 and 2014.
\end{abstract}

Keywords: Flooding, weather emergencies, hydrologic modeling.

\section{Introducción}

Las avenidas fluviales son uno de los fenómenos naturales extremos que producen las mayores consecuencias negativas para los habitantes de Uruguay. De acuerdo al Sistema Nacional de Emergencias (SINAE), autoridad nacional responsable de la gestión de las emergencias, Durazno es la segunda ciudad del país que se ve afectada periódicamente por inundaciones del río Yí. En efecto, para la inundación acaecida en mayo de 2007, la de mayor severidad registrada en Durazno, el número de evacuados ascendió a 6.000 personas, mientras que para la inundación reciente de febrero de 2010 se registraron aproximadamente 5.500 (Chreties, et al., 2011).

A nivel internacional, la Organización Meteorológica Mundial (WMO, 2011) señala que las crecidas son uno de los desastres naturales más devastadores que afectan al planeta y afirma que durante las últimas décadas la tasa de daños por crecidas ha aumentado exponencialmente. En base a ello considera que tanto el desarrollo puntual como continuo de sistemas hidrológicos de previsión y alerta son esenciales en las estrategias regionales y nacionales y constituyen un requisito para asegurar el desarrollo económico y social sustentable de las comunidades en situación de riesgo.

El estudio sistemático de la problemática de inundaciones en la ciudad de Durazno comenzó en el año 2002 con la ejecución del proyecto «Plan de emergencia para la ciudad de Durazno, República Oriental del Uruguay» (OEA, et al., 2002). En el marco de este proyecto se desarrollaron un conjunto de estudios básicos de carácter hidrológico (hidrología estadística), hidráulico, socioeconómico y de ordenamiento territorial en la zona inundable. Como resultados más significativos del proyecto se destacaron: la obtención de curvas de inundación en la ciudad para diferentes períodos de retorno de las avenidas del río Yí, el desarrollo de un primer modelo de base estadística 
de alerta temprana para la ciudad, y la consecución de acciones institucionales y técnicas que permitieron, años después, la conformación del Centro Coordinador de Emergencias Departamentales de Durazno (CECOED). Estos resultados representaron una mejora en el manejo de las emergencias de la ciudad, no obstante, diversos aspectos del plan de emergencias han requerido su revisión, actualización, mejora y, en muchos casos, la implementación de nuevas herramientas. Esto, junto con la ocurrencia en cuatro años de dos eventos extremos de alta recurrencia (mayor a 250 años), propiciaron la formulación y ejecución del «Proyecto piloto de alerta temprana para la ciudad de Durazno ante las avenidas del río Yí». Este proyecto de la red PROHIMET (ex - CYTED), financiado por la Organización Meteorológica Mundial (OMM), fue ejecutado entre 2009 y 2012 por el Instituto de Mecánica de los Fluidos e Ingeniería Ambiental (IMFIA), con la coparticipación del Instituto de Teoría y Urbanismo (ITU), la Dirección Nacional de Aguas (DINAGUA-MVOTMA), el Sistema Nacional de Emergencias (SINAE), la Administración Nacional de Usinas y Transmisiones Eléctricas (UTE), el Instituto Nacional de Meteorología (INUMET), y el Ministerio de Ganadería, Agricultura y Pesca (MGAP). El principal resultado del proyecto PROHIMET fue la creación del sistema de alerta temprana que se encuentra operativo on-line desde 2011. El mismo está basado en la modelación hidrológica-hidrodinámica del sistema cuenca-río y utiliza como información de entrada los datos de la red telemétrica de UTE así como diferentes pronósticos de precipitación en tiempo real. El usuario principal de este Sistema de Alerta es el Centro Coordinador de Emergencias Departamental de Durazno (CECOED), oficina local encargada de la gestión de las inundaciones en la ciudad.

El objetivo de este trabajo es presentar el Sistema de Alerta Temprana operativo de la ciudad de Durazno y su evaluación en el pronóstico de crecidas del río Yí para distintos tipos de tormentas ocurridas en la cuenca (corta y larga duración).

\section{Materiales y Métodos}

\section{Área de estudio}

La ciudad de Durazno se localiza en la zona centro del país,

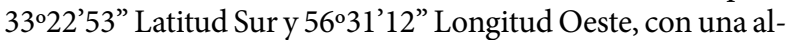
titud de $91 \mathrm{~m}$ s.n.m. La ciudad se ubica en la margen izquierda del río Yí. Aproximadamente 5.000 de sus 30.700 habitantes viven en zona de alto riesgo de inundación, la cual ocupa un área urbana de unas 500 ha.

La cuenca del río Yí tiene una superficie de $8.900 \mathrm{~km}^{2}$ aguas arriba de la ciudad de Durazno. La longitud del cauce, desde las nacientes hasta la ciudad, es de $192 \mathrm{~km}$, con una pendiente de 0,12\%, con lo que, según Kirpich (1940), resulta un tiempo de concentración de 52 horas.

\section{Red de monitoreo}

La cuenca del río Yí cuenta con una red básica de monitoreo diario de precipitación constituida por 18 estaciones convencionales operadas conjuntamente por el INUMET y UTE. Por otra parte, en las localidades de Sarandí del Yí, Polanco del Yí, Durazno y Paso del Bote, se cuenta con información histórica de niveles, y en Durazno y Sarandí del Yí se cuenta también con curva de aforos. Estas estaciones son gestionadas por la DINAGUA-MVOTMA y sus series históricas fueron utilizadas para la calibración y validación del modelo hidrológico-hidrodinámico.

Desde 2010, la cuenca dispone, además, de una red telemétrica gestionada por UTE, que incluye medición y transmisión en tiempo real de precipitación y nivel del río Yí en los siguientes sitios: Sarandí del Yí, Polanco del Yí, Durazno y Barra de Porongos. En la Figura 1 se presenta sobre el mapa de la cuenca del río Yí la localización de las estaciones convencionales de precipitación y la red telemétrica utilizada para el Sistema de Alerta Temprana.

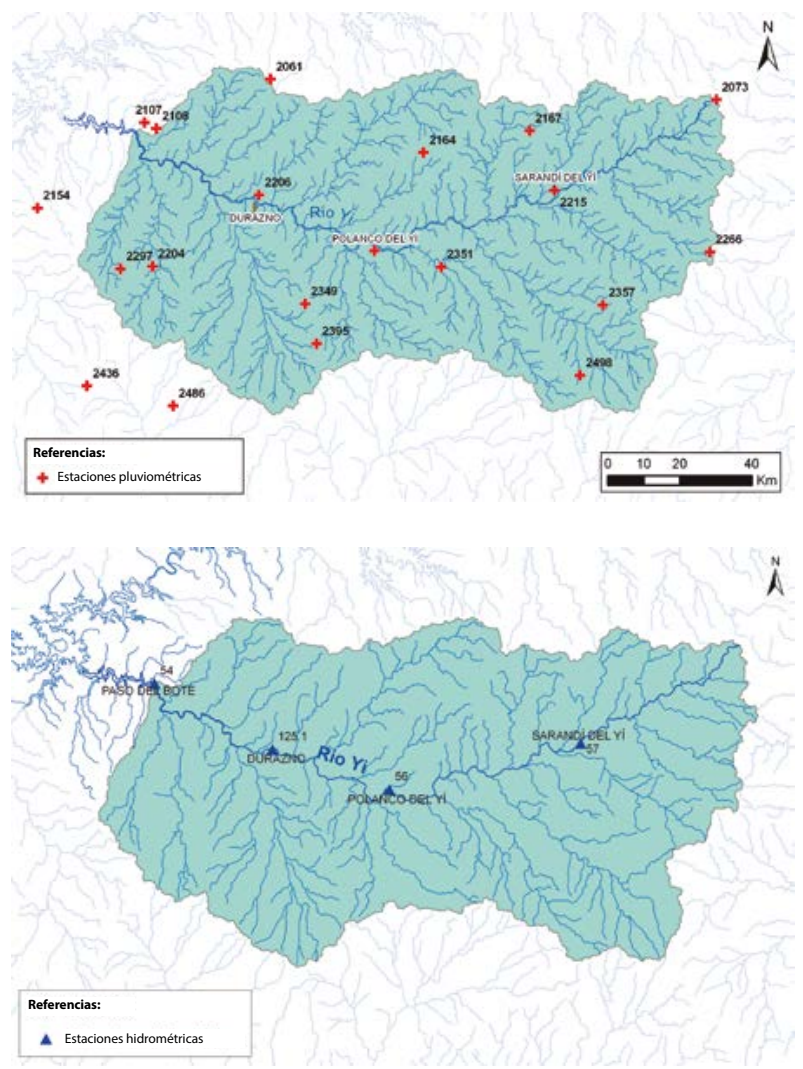

Figura 1. Localización de las estaciones pluviométricas convencionales (arriba) y estaciones hidrométricas (abajo) utilizadas en la elaboración y operación del Sistema de Alerta.

\section{Modelo hidrológico-hidrodinámico de la cuenca del río Yí}

El Sistema de Alerta Temprana se apoya en un modelo hidrológico-hidrodinámico de la cuenca del río Yí que constituye el elemento técnico central en su operación. El esquema general del modelo puede observarse en la Figura 2 (izquierda) y se basa en la discretización de la cuenca en 16 sub-cuencas de aporte al río Yí, cuya superficie promedio es $800 \mathrm{~km}^{2}$. En cada una de esas 16 sub-cuencas se implementó un modelo hidrológico de tipo concentrado, basado en el método propuesto por el Natural Resourses Conservation Service (USDA, 2010). Se utilizó información de clasificación de suelos disponible en la Carta de Reconocimiento de Suelos de Uruguay escala 1:1000000 (MGAP, 1979) y en (Molfino, et al., 2001) y en base a ella se elaboró un mapa con la distribución espacial de los grupos hidrológicos presentes en la 

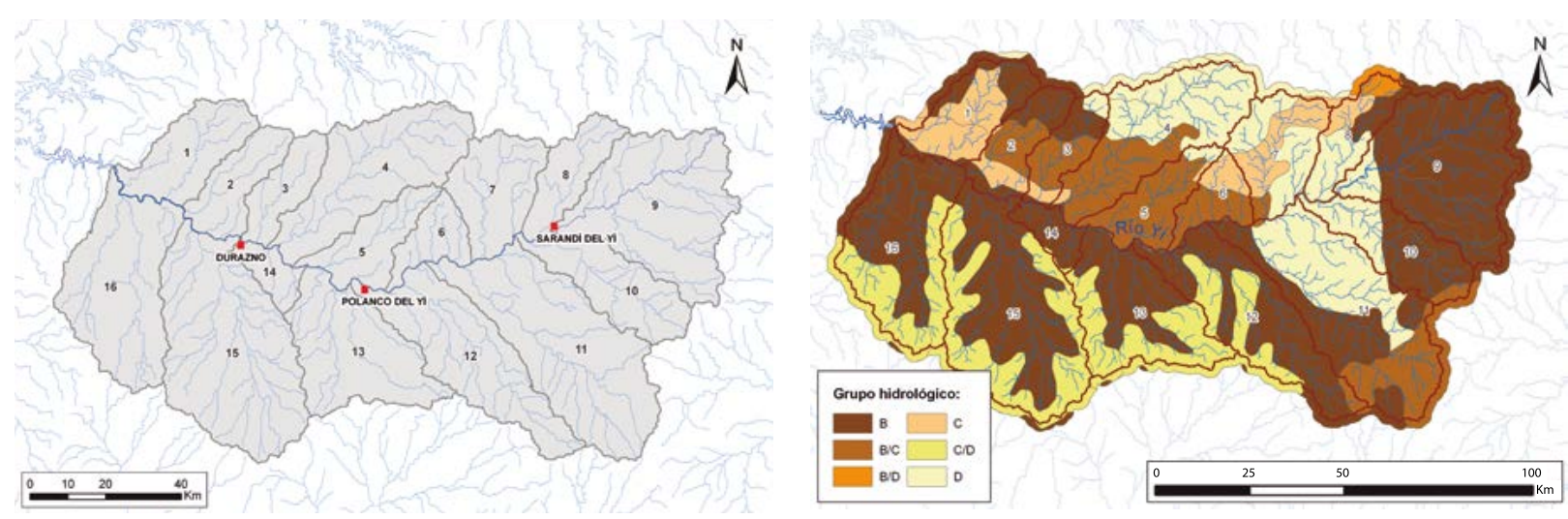

Figura 2. Esquema de modelación. Discretización en 16 sub-cuencas (izquierda). Distribución espacial del Grupo Hidrológico según la clasificación del NRCS.

cuenca (Figura 2, derecha). Posteriormente, incorporando información satelital de uso de suelo de la cuenca, se obtuvo el valor del parámetro Número de Curva en cada sub-cuenca. La respuesta de cada sub-cuenca se modeló considerando el método del hidrograma unitario triangular-HUT (NRCS, 2010), estimando el tiempo de concentración mediante la formulación de Tucci (Tucci, 2000), que incorpora la pendiente media de la cuenca y el número de curva como parámetros fundamentales.

El resultado del modelo hidrológico en cada una de las 16 sub-cuencas es un hidrograma correspondiente al evento extremo de precipitación que se esté considerando. Asociado al modelo hidrológico fue implementado un modelo hidrodinámico para el río Yí, entre Sarandí del Yí y Paso
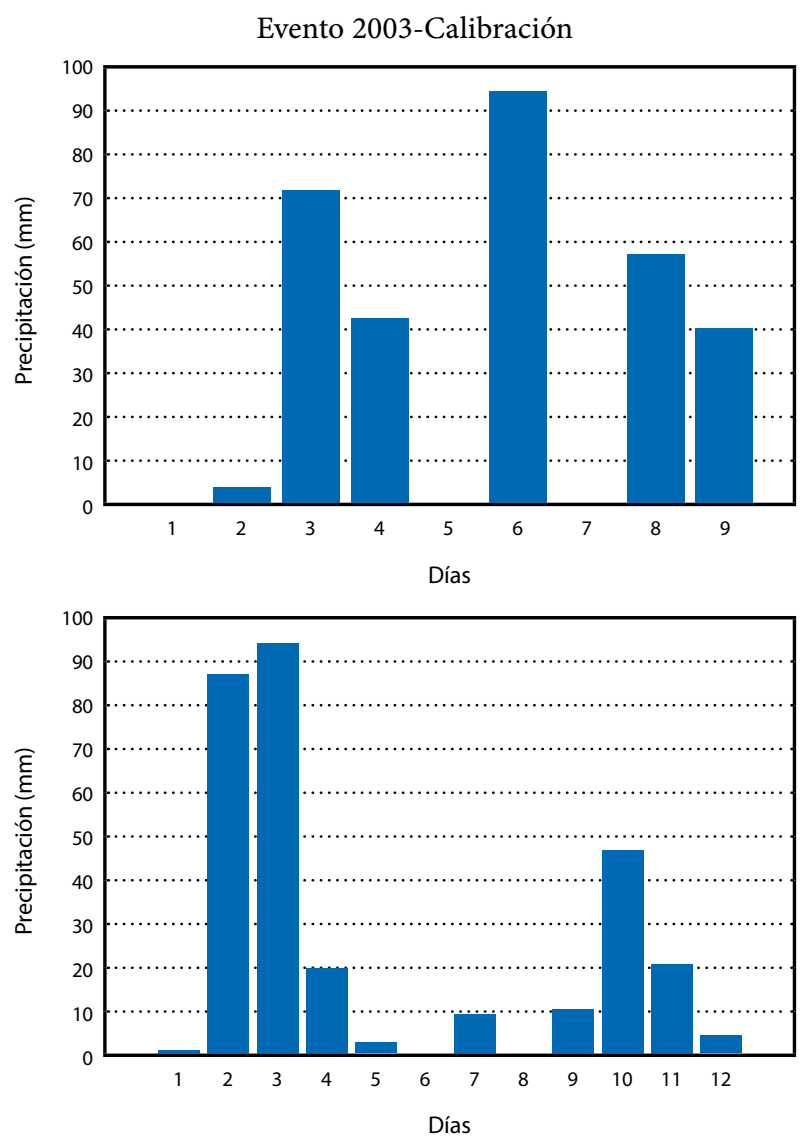

del Bote, que toma los resultados del modelo hidrológico y resuelve el tránsito hidrodinámico de la onda de crecida por el río. El modelo hidrodinámico utilizado (Hec-Ras 4.1; USACE, 2010) permite la resolución de las ecuaciones de Saint-Venant 1D en estado no-estacionario en base a un esquema numérico de diferencias finitas implícito. Para su implementación se utilizó información topo-batimétrica de 40 secciones transversales al río, que fueron relevadas en su mayoría en el marco del proyecto PROHIMET, así como todas las obras de drenaje de primera importancia, como puentes y alcantarillas.

Como condición de borde aguas arriba del modelo hidrodinámico se fijó el hidrograma en Sarandí del Yí (obtenido del modelo hidrológico de esta sub-cuenca). Como condición de

Evento 2005-Calibración
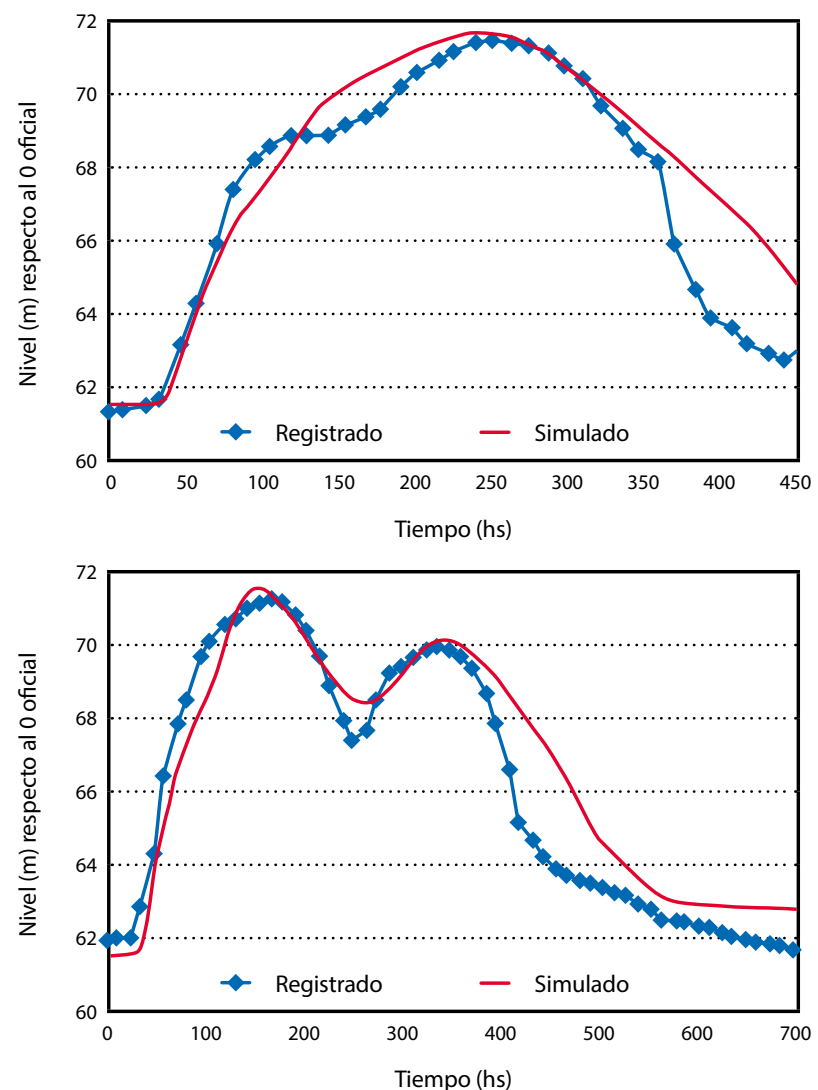

Gráfico 1. Calibración del modelo hidrológico-hidrodinámico. 
Evento 2007-Calibración
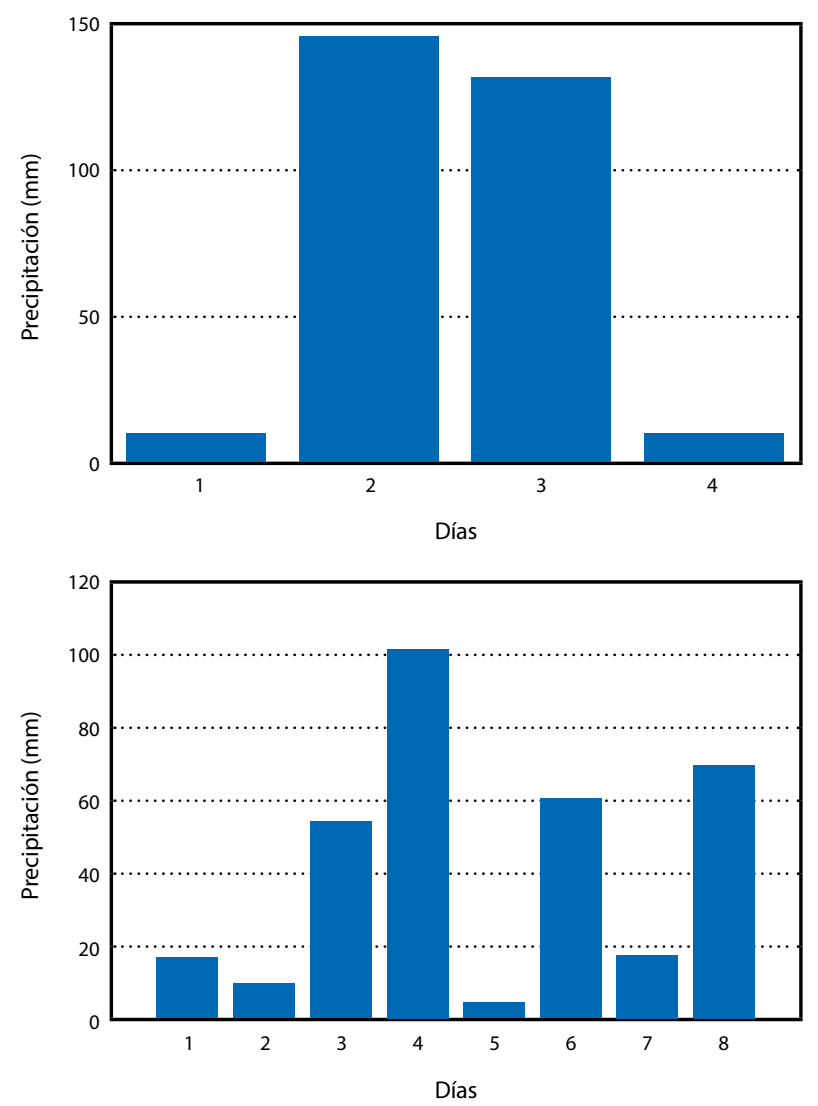

Evento 2010-Validación
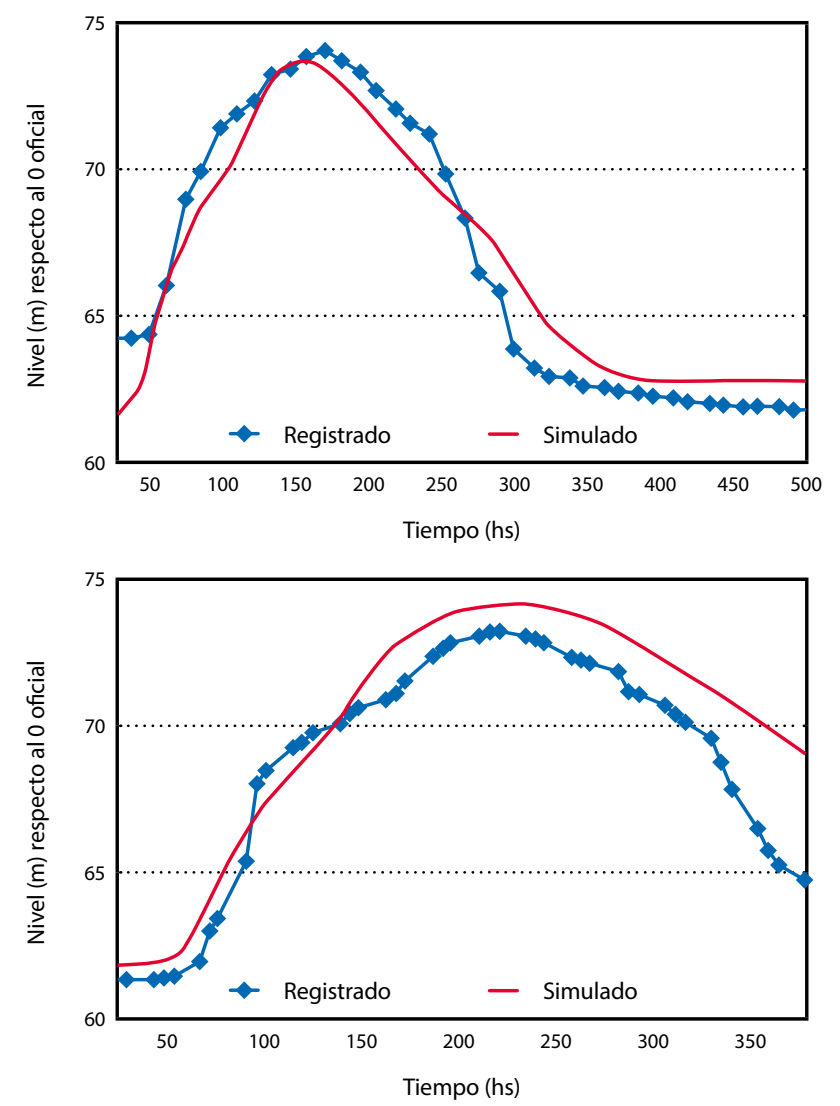

Gráfico 2. Calibración y validación del modelo hidrológico-hidrodinámico.

borde aguas abajo se verificó que el río 20 kilómetros aguas abajo de Durazno alcanzara un régimen uniforme, siendo esta la condición de borde fijada. Finalmente, los restantes hidrogramas generados en las sub-cuencas se incorporaron al modelo hidrodinámico como aporte lateral de caudal no estacionario. Sobre esa base, dado un evento extremo de precipitación (hietograma observado o pronosticado), el modelo hidrológico-hidrodinámico permite obtener en cada zona de la ciudad el nivel máximo y el tiempo de ocurrencia del evento extremo.

\section{Calibración y validación del modelo hidrológico-hidrodinámico}

Una vez implementado el modelo hidrológico-hidrodinámico, sus parámetros del fueron calibrados y validados en base a información observada de eventos extremos pasados. Para la calibración se seleccionaron los eventos de 2003, 2005 y 2007, ajustando los siguientes parámetros del modelo hidrológico-hidrodinámico: NC (número de curva), tc (tiempo de concentración) y $\mathrm{n}$ (coeficiente de rugosidad de Manning). Como funciones objetivo para el ajuste se consideró minimizar la diferencia entre el nivel máximo observado en Durazno y el nivel máximo modelado, y simultáneamente minimizar la diferencia de volumen de escorrentía (observado y modelado) para todo el evento. Los valores finales del coeficiente de rugosidad en cauce y planicie (en todo el tramo modelado) que minimizan ambas funciones objetivo son 0.080 y $0.150 \mathrm{~s} / \mathrm{m}^{(1 / 3)}$, respectivamente. El tiempo de concentración calculado en base a la formulación original de Tucci se mantuvo en los valores teóricos, mientras que el Número de Curva resultante en cada cuenca es un valor incrementado un $20 \%$ del valor teórico.

Luego del proceso de calibración, una vez conseguidos los valores de los parámetros del modelo se procedió a analizar su desempeño frente a un evento extremo diferente (febrero de 2010), lo que constituyó la validación del modelo. En los Gráficos 1 y 2 se presentan los resultados de la calibración y validación del modelo para cada uno de los eventos seleccionados. Mayores detalles de los resultados de la calibración del modelo se pueden consultar en Silveira, et al., 2012.

\section{Operación del Sistema de Alerta Temprana}

La puesta en operación del Sistema de Alerta Temprana incluyó la incorporación en tiempo real de los datos de precipitación registrados por las estaciones telemétricas de UTE, así como de dos modelos de pronóstico meteorológico: WRF (ejecutado en IMFIA) y los resultados del modelo COSMO-INMET-Brasil. Se realiza en forma automática y diaria una simulación del modelo hidrológico-hidrodinámico en base a los datos registrados hasta la fecha de simulación y pronosticados por ambos modelos meteorológicos, obteniéndose como resultado la evolución temporal de los niveles del río Yí en Durazno, desde el momento de la simulación hasta el final de la crecida pronosticada. Los resultados son visualizados en una página web junto con la precipitación observada, pronosticada y los niveles observados en las estaciones telemétricas del río Yí (ver página web sugerida). 


\section{Resultados}

El modelo hidrológico-hidrodinámico, que constituye la base del sistema para previsión temprana de niveles del río Yí frente a la ciudad de Durazno, fue calibrado y validado para eventos extremos habituales, con una duración de entre 1 y 7 días. No obstante, a continuación se ilustra la performance frente a un evento atípico, caracterizado como un bloqueo atmosférico. La lluvia, que se inició el 21 de enero y continuó hasta el 11 de febrero de 2014, fue provocada por un sistema frontal; esto es, la unión de dos masas de aire de distinta temperatura, una cálida proveniente del sur de Brasil y otra fría al sur de Uruguay, que quedó estacionada sobre el Río de la Plata, provocando precipitaciones continuas sobre Buenos Aires y el sur de Uruguay. Este tipo de fenómenos normalmente no dura más de 24 horas y desaparece del territorio nacional, según afirmación de Mario Bidegain (El Observador, 2014). En este caso, la persistencia fue de entre 15 a 21 días.

Entre el 21 y el 27 de enero se produjeron lluvias leves, con intermitencias. El nivel de alerta previsto por el SATI-Uy cambió de verde a amarillo el martes 28 de enero, con base en la lluvia prevista por los pronósticos a cinco días. El Gráfico 3 muestra la previsión del SATI-Uy, con indicación de niveles máximos pronosticados en los puentes sobre el río Yí, denominados

\section{Martes 28/1/2014 Nivel amarillo:}

Nivel Máximo Pronosticado Puente Nuevo: 9,24 m. Nivel Máximo Pronosticado Puente Viejo: 8,21 m.

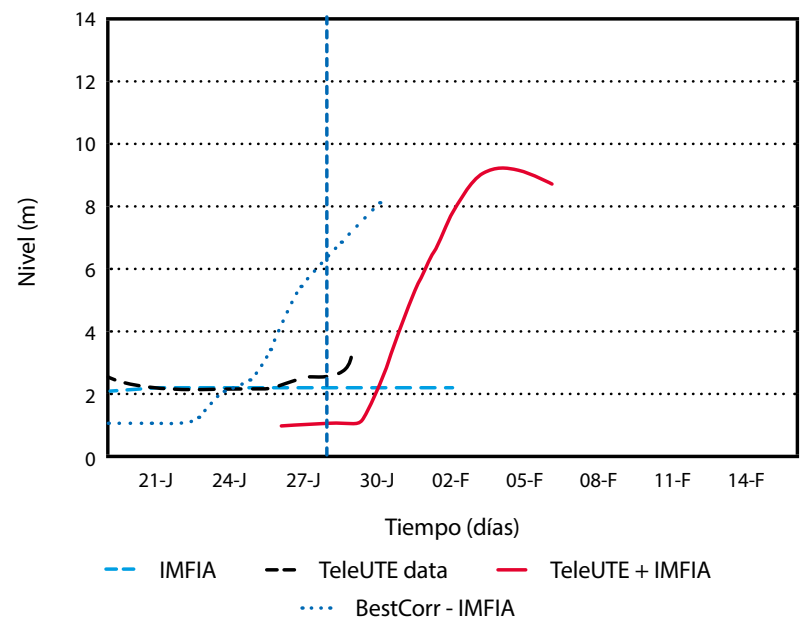

Fechas esperadas Nivel máx.: 2014-02-03 al 05 Precipitación Acumulada/Pronosticada: $127 \mathrm{~mm}$

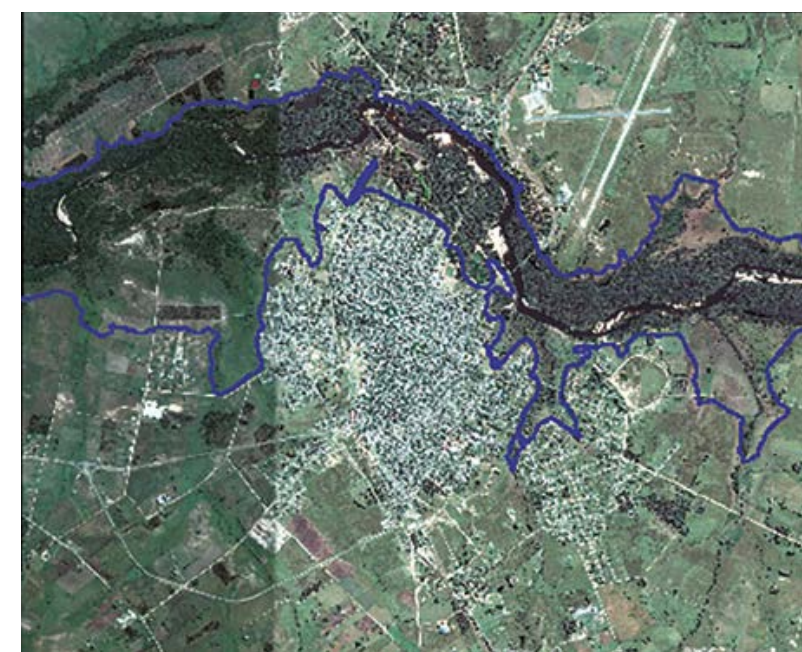

Gráfico 3. Previsión del Sistema de Alerta Temprana de Inundaciones, emitida el 28 de enero de 2014.

Miércoles 29/1/2014 Nivel rojo:

Nivel Máximo Pronosticado Puente Nuevo:10,61 m. Nivel Máximo Pronosticado Puente Viejo: 9,48 m.

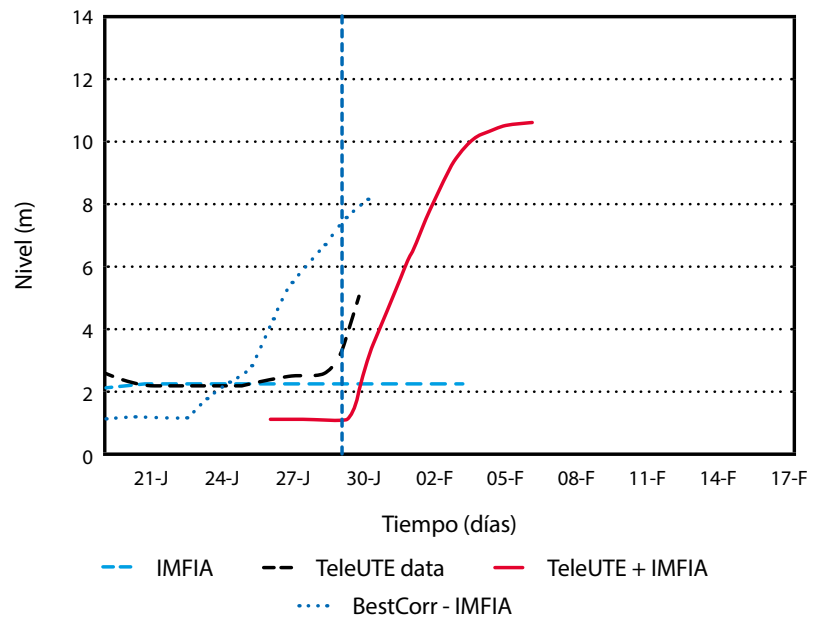

Fechas esperadas Nivel máx.: 2014-02-05 al 07 Precipitación Acumulada/Pronosticada: $204 \mathrm{~mm}$

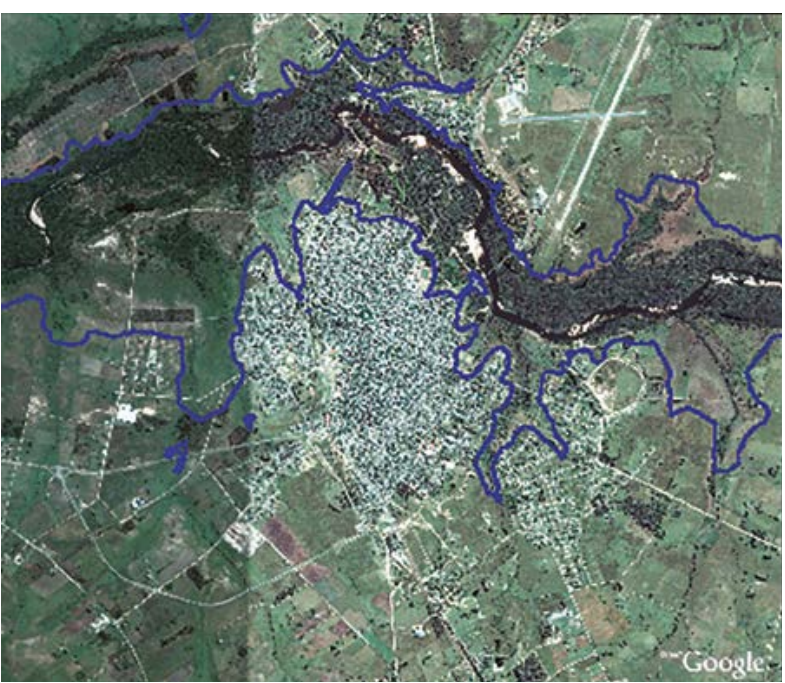

Gráfico 4. Previsión del Sistema de Alerta Temprana de Inundaciones, emitida el 29 de enero de 2014. 


\section{Jueves 6/2/2014 Nivel rojo}

Nivel Máximo Pronosticado Puente Nuevo:12,06 m. Nivel Máximo Pronosticado Puente Viejo: 10,83 m.

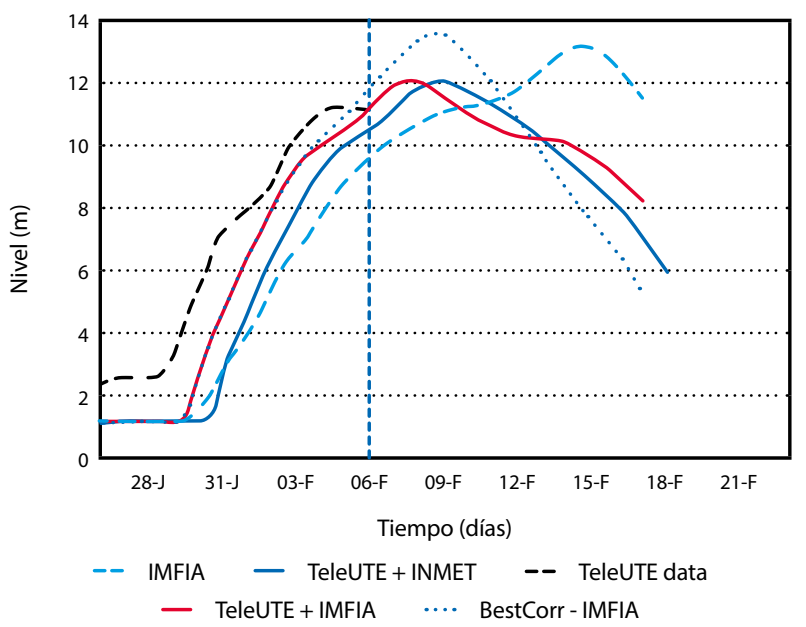

Fechas esperadas Nivel máx: 2014-02-08 al 10

Precipitación Acumulada/Pronosticada: $366 \mathrm{~mm}$

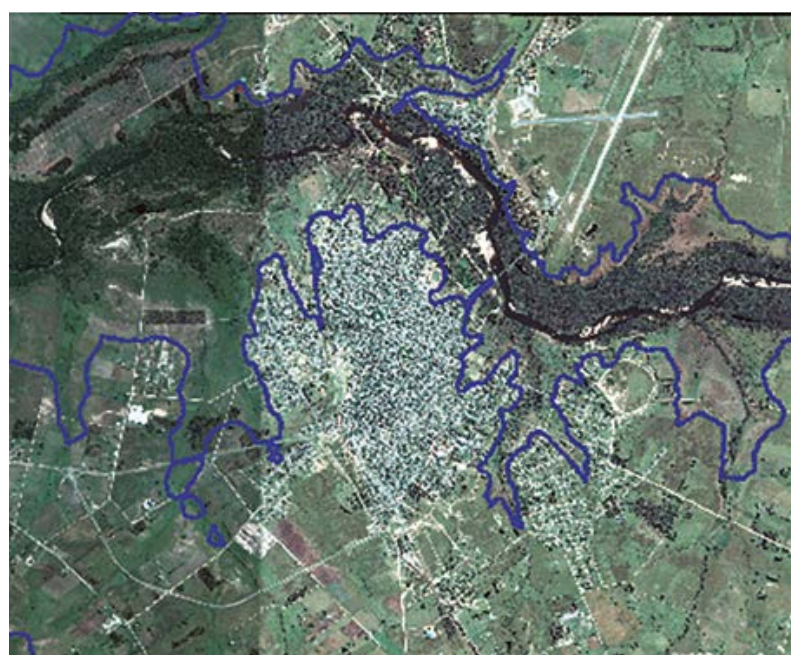

Gráfico 5. Previsión del Sistema de Alerta Temprana de Inundaciones, emitida el 6 de febrero de 2014.

"nuevo», en ruta 5, donde está ubicada la estación telemétrica de UTE, y «viejo», por constituir la referencia con que los duraznenses caracterizan la gravedad de la inundación. Asimismo, se indica la fecha esperada para la ocurrencia del nivel máximo y la suma de la precipitación acumulada registrada desde el inicio del evento más la pronosticada para los cinco días subsiguientes. Los gráficos muestran la evolución del nivel del río (nivel registrado por la estación telemétrica, punteado en negro, y el previsto por el SATI-Uy, línea llena en rojo), y en el plano se muestra la zona que se verá afectada por el desborde del río Yí al alcanzarse el nivel máximo pronosticado.

$\mathrm{Al}$ persistir el bloqueo atmosférico, la suma de precipitación acumulada y pronosticada evolucionó a $204 \mathrm{~mm}$, en comparación con la lluvia promedio mensual de $103 \mathrm{~mm}$, con lo que la alerta emitida por el SATI-Uy evolucionó a nivel rojo, según puede observarse en el Gráfico 4 . En él puede observarse el buen acompañamiento de la rama ascendente del hidrograma (niveles observado y pronosticado), con un desfasaje resultante de las condiciones iniciales que asume el modelo.

El domingo 2 de febrero persistía el nivel rojo, con una precipitación acumulada/pronosticada de $505 \mathrm{~mm}$, que el lunes 3 fue corregida a $436 \mathrm{~mm}$ y el jueves 6 a $366 \mathrm{~mm}$, en función de la lluvia pronosticada. El Gráfico 5 muestra que se mantuvo el buen ajuste de la rama ascendente del hidrograma (niveles observados y pronosticados). Finalmente, la precipitación total registrada fue de $362 \mathrm{~mm}$, y el nivel máximo que se alcanzó en «puente nuevo» fue 11,23. Es decir, 0,83 m menor a lo previsto por el SATI-Uy, error que se considera razonable en virtud de las incertidumbres en la información relevada para su operación. En total fueron evacuadas 2.290 personas. El Gráfico 6 presenta la evolución del evento extremo atípico, que se extendió desde el 28 de enero al 11 de febrero de 2014.

Además del evento atípico descripto anteriormente, en 2011 se gestionaron tres eventos de 2 a 4 días de duración. En la Tabla 1 se compara el nivel máximo previsto por el SATI-Uy con el nivel máximo observado, así como la ocurrencia del máximo. Como puede observarse, el error en la previsión fue inferior a 0,5 metros en los tres eventos y la ocurrencia del máximo presentó una diferencia máxima de 16 horas.

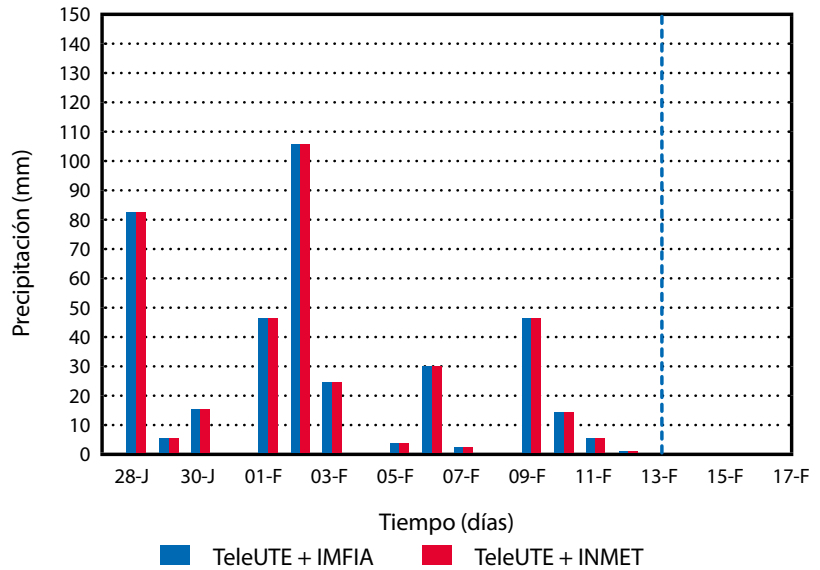

Gráfico 6. Hietograma de lluvia del evento meteorológico que tuvo lugar en la cuenca del río Yí entre el 28 de enero y el 11 de febrero de 2014 y que alcanzó una precipitación acumulada de $366 \mathrm{~mm}$.

\section{Discusión}

Previo al desarrollo del SATI-Uy, la ciudad de Durazno disponía de un modelo estadístico denominado «Cascos Blancos», que permitía prever el nivel máximo que puede alcanzar el río Yí frente a la ciudad, con base en los registros diarios de precipitación acumulada en cuatro pluviómetros y el nivel máximo en Sarandí del Yí (Figura 3), datos que se ingresaban en una ecuación de regresión múltiple. Este es un modelo de muy simple aplicación. No obstante, pueden señalarse las siguientes desventajas: i) en la fase operativa, durante la ocurrencia del evento, no se conoce el nivel máximo que puede alcanzar el río Yí frente a Sarandí del Yí; ii) la lluvia total en cada pluviómetro solo puede conocerse a las 7 a.m. del día siguiente, y iii) no proporciona la fecha de ocurrencia del máximo, ni otorga información sobre la permanencia 


\begin{tabular}{|c|c|c|c|c|c|c|}
\hline \multirow{2}{*}{ Evento } & \multicolumn{2}{|c|}{ Previsión del SATI-Uy } & \multicolumn{2}{c|}{ Observado } & \multicolumn{2}{c|}{ Diferencia de la previsión (m) } \\
\cline { 2 - 7 } & $\begin{array}{c}\text { Nivel máximo } \\
(\mathbf{m})\end{array}$ & $\begin{array}{c}\text { Fecha de } \\
\text { ocurrencia }\end{array}$ & $\begin{array}{c}\text { Nivel } \\
\text { máximo }(\mathbf{m})\end{array}$ & $\begin{array}{c}\text { Fecha de } \\
\text { ocurrencia }\end{array}$ & $\begin{array}{c}\text { Nivel } \\
(\mathbf{m})\end{array}$ & $\begin{array}{c}\text { Ocurrencia } \\
(\mathbf{h s})\end{array}$ \\
\hline $24-25 / 05 / 2011$ & 6,64 & $28 / 0505: 00$ & 6,20 & $27 / 0513: 00$ & $+0,44$ & +16 \\
$16-19 / 06 / 2011$ & 6,42 & $21 / 0618: 00$ & 6,74 & $21 / 0618: 00$ & $-0,32$ & 0 \\
$15-16 / 07 / 2011$ & 8,30 & $20 / 0710: 00$ & 8,62 & $20 / 0710: 00$ & $-0,32$ & 0 \\
\hline
\end{tabular}

Tabla 1. Comparación de niveles y tiempos pronosticados y observados para tres eventos extremos de corta duración gestionados por el CECOED en base al SATI-Uy.

de niveles por encima de cotas críticas. Con este sistema se desconocía, entre otros, cuánto tiempo se disponía para la evacuación de personas y bienes, el tiempo que permanecerían en refugios, la duración del corte de vías de transporte. Por lo tanto, resultaba muy difícil realizar una programación y/o previsión de los costos, alimentos, combustible y demás insumos requeridos para atender a la población durante la emergencia. En cambio, el SATI-Uy maneja información horaria en tiempo real y datos de pronósticos meteorológicos a efectos de incrementar el tiempo de alerta para gestionar la emergencia, proporciona información sobre fecha y hora de ocurrencia del nivel máximo y sobre la forma del limnigrama y, por consiguiente, otorga información sobre la permanencia de niveles por encima de cotas críticas de inundación, que es de sumo valor para planificar y gestionar la emergencia.

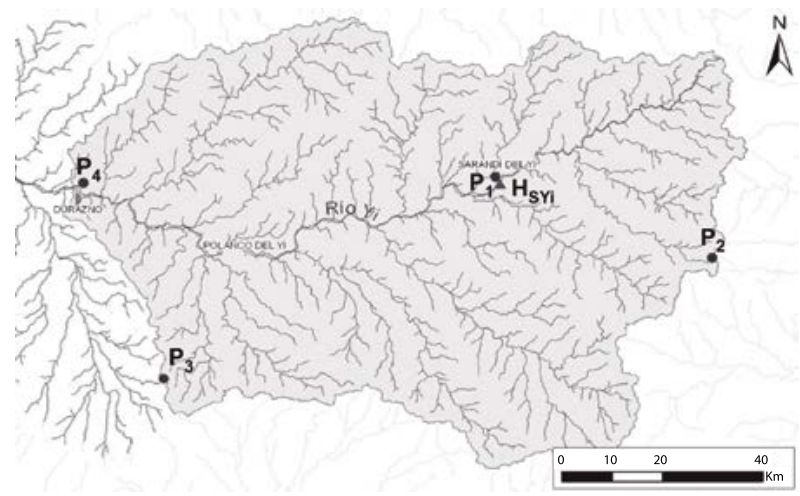

Figura 3. Información de base del modelo estadístico «Cascos Blancos», de previsión de niveles máximos del río Yí frente a la ciudad de Durazno.

\section{Conclusiones}

En este trabajo fueron presentadas las principales características del Sistema de Alerta Temprana ante Inundaciones que se encuentra operativo para la ciudad de Durazno desde el año 2011. A su vez, se mostraron los resultados de su desempeño frente a eventos de corta duración (más habituales) así como eventos diferentes a los registrados en el pasado (bloqueo atmosférico). En todas las situaciones, los resultados fueron muy satisfactorios desde el punto de vista técnico, ya que las diferencias ocurridas entre los niveles pronosticados y lo realmente ocurrido están dentro del error habitual para este tipo de sistemas fluviales. Más allá de los resultados del desempeño puramente técnico, resulta de sumo interés analizar los resultados del SATI-Uy para el CECOED, organismo encargado de gestionar las emergencias en el departamento. En este sentido, la experiencia del CECOED ha sido positiva, y se ha destacado en varias oportunidades que el disponer de un Sistema de Alerta Temprana de Inundaciones ha permitido gestionar las emergencias con mayor eficiencia, evitando tener que evacuar a los damnificados en horas de la noche y que las aguas afecten sus bienes. Asimismo, ha hecho posible planificar la infraestructura necesaria para atender la emergencia, por ejemplo, el número de camiones, carpas, alimentos y abrigo que será necesario proporcionar.

\section{Reconocimientos}

El desarrollo del Sistema de Alerta Temprana de Inundaciones (SATI-UY) fue financiado por la Organización Meteorológica (OMM) con sede en Ginebra, Suiza, como proyecto piloto que fuera presentado por las instituciones nacionales a la red PROHIMET (Red Iberoamericana para el monitoreo y previsión de fenómenos hidrometeorológicos). El equipo de investigadores del Instituto de Mecánica de los Fluidos e Ingeniería Ambiental (IMFIA), de la Facultad de Ingeniería, Universidad de la República, recibió el apoyo y cooperación de numerosas instituciones públicas vinculadas al monitoreo hidrometeorológico y las emergencias nacionales, así como de la municipalidad de Durazno.

\section{Referencias}

El Observador, 2014. Llovió en un día lo que llueve en un mes [En línea]. Montevideo: El Observador. [Consulta: 10 de febrero de 2015]. Disponible en: http://www.elobservador. com.uy/noticia/271202/en-lavalleja-llovio-en-un-dia-loque-habitualmente-llueve-en-un-mes/

Chreties, C., Silveira, L., López G. y Crisci, M., 2011. Sistema de alerta temprana para la ciudad de Durazno, Uruguay. En: Memorias del V Simposio regional sobre hidráulica de ríos-Ríos 2011. Santiago del Estero: [s.n.].

Kirpich, Z.P., 1940. Time of concentration of small agricultural watersheds. En: Civ Eng, 1940, 10(6), pp.362.

MGAP, 1979. Carta de reconocimiento de suelos del Uruguay. Vol. 111. Montevideo: Ministerio de Ganadería, Agricultura y Pesca. Dirección de Suelos y Aguas.

Molfino, J.H., Califra, A., 2001. Agua disponible de las tierras del Uruguay. Montevideo: Ministerio de Ganadería Agricultura y Pesca.

Natural Resourses of Conservation Service (NRCS), 2010. National engineering handbook-210-630-hydrology. 
Washington: United States Department of Agriculture.

OEA, BID, CB, MTOP, 2002. Plan de emergencia para la ciudad de Durazno, República Oriental del Uruguay. Montevideo: OEA.

Rodríguez, J. M. 2014. Río Yí crece 10 centímetros por hora [En línea]. Montevideo: El Observador. [Consulta: 10 de febrero de 2015]. Disponible en: http://www. elobservadormas.com.uy/noticia/2014/02/02/4/rio-yicrece-10-centimetros-por-hora_270752/

Silveira, L., López G., Chreties C., y Crisci, M., 2012. Steps towards an early warning model for flood forecasting in Durazno city in Uruguay. En: Journal of Flood Risk Management, 5(3), pp.270-280.

Tucci, C. E.M., 2000. Hidrologia: ciencia e aplicaçao. 2da. ed., 1a reimp. Porto Alegre: Ed. Universidade, UFRGS: ABRH, 2000. (Coleçao ABRH de Recursos Hídricos, 4.) ISBN 85-7025-298-6.

US Army Corps of Engineers, 2011. HEC-RAS 4.1 Hydraulics Manual. Washington: US Army.

USDA, 2010. Part 630 Hydrology -National Engineering Handbook (NEH). Washington: Natural Resources Conservation Service, U. S. Departament of Agriculture. WMO, 2011. Manual on flood forecasting and warning. Ginebra: WMO. (WMO, 1072). ISBN 978-92-63-11072-5.

Página web sugerida

http://www.fing.edu.uy/imfia/prohimet/Prohimet-Yi/Durazno/Global/data/2014-01-28/WebOutput.htm 